\title{
Association of Cardiovascular Fitness and Metabolic Syndrome in Male Police Officers
}

\author{
MATTHEW S. SCHMIDT, STEVEN E. MARTIN, BRAD S. LAMBERT, ALEX
}

CARRADINE, JOHNS. GREEN, and STEVE F. CROUSE

Applied Exercise Science Lab; Department of Health and Kinesiology; Texas A\&M University; College Station, TX

\section{Category: Undergraduate}

Metabolic syndrome has been shown in numerous studies to be related to a higher incidence of coronary artery disease (CAD). A study by Jurca and coworkers (Med. Sci. Sports Exerc 36(8):1301-1307, 2004) found a relationship between aerobic fitness and the prevalence of metabolic syndrome in a group of men enrolled in the Aerobics Center Longitudinal Study (ACLS). In addition, recent work in our lab (Int J Exerc Sci 2(1): S43, 2009) has supported these findings. However, research exploring this relationship is still lacking in male police officers. Previous research has suggested that the prevalence of CAD in police officers may be higher than in the general population. Purpose: To further evaluate the association of metabolic syndrome and cardiovascular fitness in male police officers. Methods: As part of an annual physical exam, 51 male fire fighters (average age $=39$ ) underwent evaluation of risk factors associated with metabolic syndrome as defined by NCEP III. These include the presence of three or more of the following: Waist Circumference $>40 "$, HDL Cholesterol $<40 \mathrm{mg} / \mathrm{dL}$, Triglycerides $>150 \mathrm{mg} / \mathrm{dL}$, Blood Glucose $>110 \mathrm{mg} / \mathrm{dL}$, and Resting Blood Pressure $>130 / 85 \mathrm{~mm} \mathrm{Hg}$. Aerobic Fitness was determined by estimating VO2max from time on treadmill during a Bruce protocol. Results: The subjects were ranked and divided into quartiles based on VO2max. All data were analyzed using a Chi Square test $(\mathrm{p}<0.05)$. Prevalence of metabolic syndrome increased across quartiles as cardiovascular fitness declined. Conclusion: These data suggest that as cardiovascular fitness improves, the likelihood of male police officers having metabolic syndrome decreases.

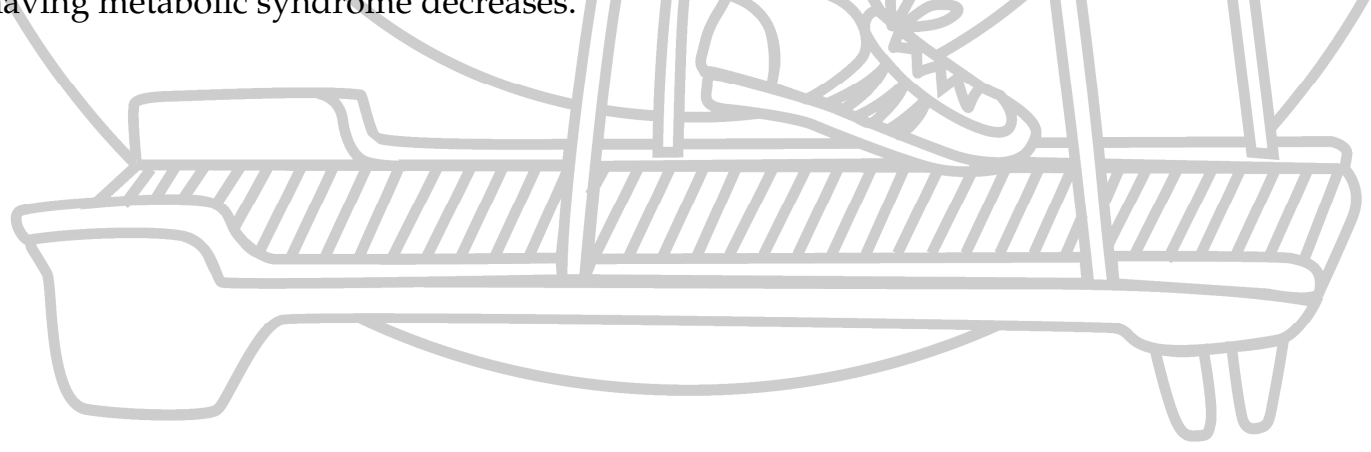




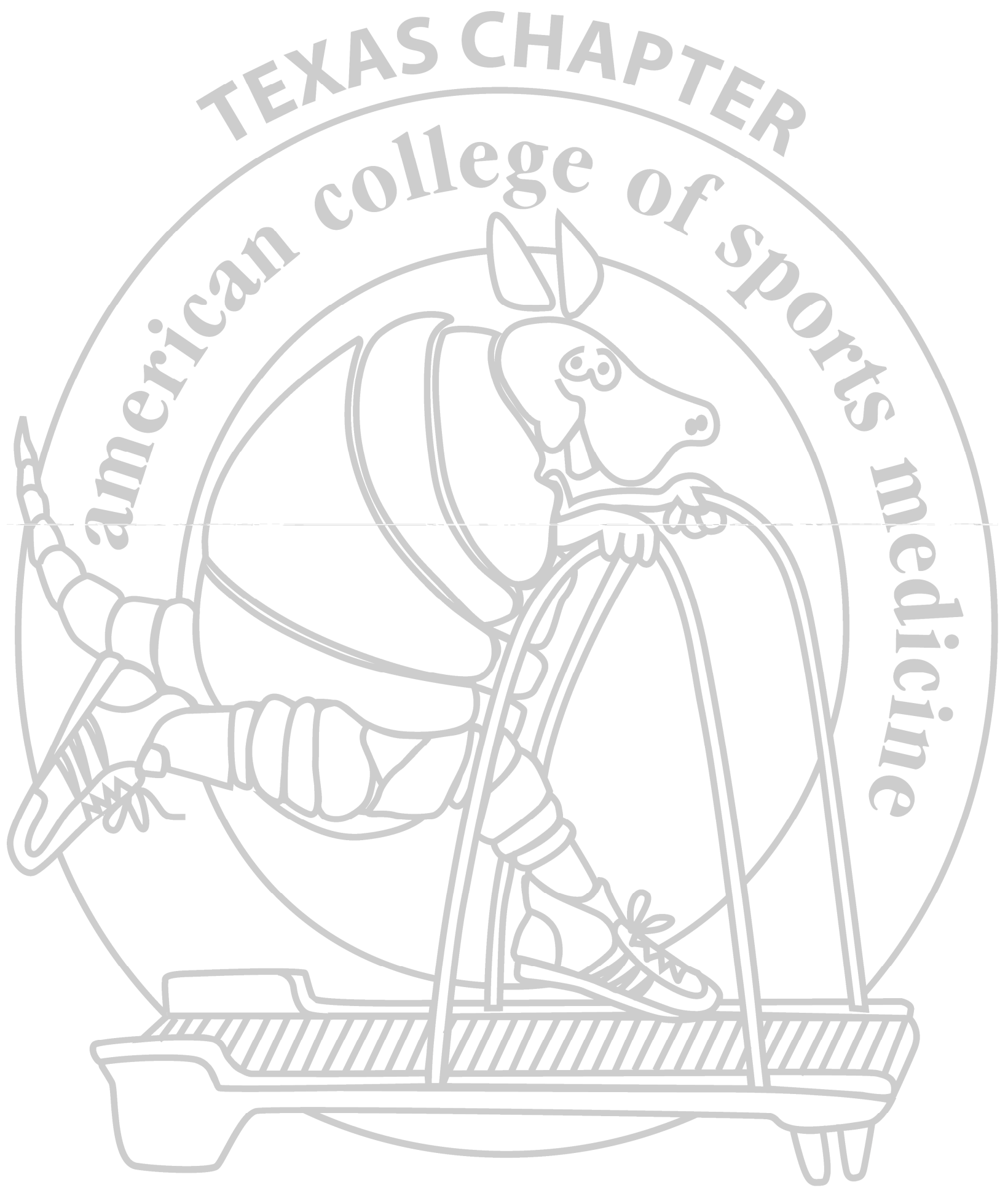

International Journal of Exercise Science

www.tacsm.org 